
\title{
\& Research Square \\ Highly sensitive biomarker biosensor for early detection of cancer biomarkers
}

\section{Jaydev Upponi}

Northeastern University

Tiziana Musacchio

Northeastern University

Salome Siavoshi

Northeastern University

Asanterabi Malima

Northeastern University

Cihan Yilmaz

Northeastern University

William C. Hartner

Northeastern University

Tatyana Levchenko

Northeastern University

Sivasubramanian Somu

Northeastern University

Vladimir P. Torchilin ( $\nabla$ v.torchilin@northeastern.edu )

Northeastern University

Ahmed Busnaina

Northeastern University

\section{Research Article}

Keywords: Tumor biomarkers, biosensors, chips, antigens, nucleosome, carcinoembryonic antigen, ELISA

Posted Date: February 25th, 2021

DOI: https://doi.org/10.21203/rs.3.rs-238414/v1

License: (9) This work is licensed under a Creative Commons Attribution 4.0 International License.

Read Full License 


\section{Abstract}

Biomarkers are emerging as potentially important diagnostic tools for cancer and many other diseases. However, many current detection systems for suffer from insufficient sensitivity. To address this concern, we developed a highly sensitive biosensor, featuring monoclonal antibody-coated polystyrene nanobeads assembled in the trenches of a microchip, for the detection of cancer biomarkers. These biosensors detected nucleosomes and carcinoembryonic antigen in serum at concentrations of 62.5 and 15.6 $\mathrm{pg} / \mathrm{mL}$, respectively. Very low detection limits that suggest such devices might be beneficial for the early detection of tumors and for monitoring of patients in remission.

\section{Introduction}

The idea for implementing biosensors was born in the 1960s with the pioneering work of Clark and Lyons ${ }^{1}$. In general, a biosensor is a device featuring a biological sensing element that transforms a biological event into a signal that can then be interpreted. The recognition of a biomolecule or biomarker should be highly selective, with the sensing element being immobilized through physical adsorption, entrapment, or covalent attachment ${ }^{2-4}$. Biomarkers are important diagnostic and monitoring tools for cancer and many other diseases. Capturing minute concentration levels of biomarkers in serum or blood is extremely crucial for the early detection, monitoring, treatment and management of a disease and should be easy to detect and measurable across populations ${ }^{5}$. Biomarkers assist not only in the early cancer detection and the development of preventive treatments but also in the identification of new targets for therapy ${ }^{6}$. It also assists in the making of suitable clinical decisions, and helping to detect the reoccurrence of cancer following treatment ${ }^{7,8}$. Among the many available biomarkers, NS and CEA are particularly useful for the detection of various cancers and for monitoring the effectiveness of cancer treatment ${ }^{9} 10,11$.

Recently, various new approaches have been developed for sensitive biomarker detections, including nanowire sensor arrays, rolling circle amplification DNA, nanocantilevers, and nanoparticle-based biobarcodes ${ }^{12-15}$. However, these biosensors could be further improved through the addition of features such as remote testability, high reliability, ease of use, rapid screening and early detection that contribute to the cost of manufacturing ${ }^{16}$. The enzyme-linked immunosorbent assays (ELISA) kits are the most commonly used techniques for the detection of biomarkers. Although these assays combine easy sample handling with flexible and simple analyses, they are expensive, laborious, and time-consuming, and often lack sufficiently high sensitivity and specificity ${ }^{16,17}$.

Many of the conventional antibody/antigen-based ELISA-based assays are highly specific, however, they lack the level of sensitivity needed for detection of disease at an early stage. One of the main reasons is poor or insufficient alignment and distribution of the antibodies on the sensor substrate that limits the number of antibody-antigen binding events ${ }^{18}$. To address this challenge nanoparticles (NPs) have been used to effectively in regulate the alignment and distribution of antibodies on its surface to enhance sensitivity and improve interaction with the substrate. ${ }^{15,19,20}$. A particularly interesting recent advance in 
biomarker detection is the use of antibody arrays with the capacity to detect biomarkers at concentrations below current detection limits without modifying the simplicity of the ELISA technique itself ${ }^{21}$.

The detection of very low concentrations of cancer biomarkers can be performed using immunosensors as good alternatives to traditional immunoassays. Taking full advantage of the materials and processing tools used for microfabrication, we have developed an ELISA-based highly sensitive SU-8 epoxy-based negative photoresist biosensor assembled with monoclonal antibody (mAb) 2C5- or CEA-coated PSL nanoparticles assembled into trenches on a microchip for detection of NS and CEA respectively.

\section{Results}

\section{Immobilization and characterization of antibody coated nanobeads}

mAb 2C5, mAb CEA, or the control, nonspecific IgG were adsorbed onto the PSL nanobeads (Fig.1a) to form a monolayer of protein on the surfaces of nanobeads ${ }^{22,23}$. Regardless of the type of antibody, the adsorption yield was greater than $50 \%$ in all cases, as estimated using the bicinchoninic acid (BCA) assay. The size and zeta potential of the nanobeads were crucial parameters affecting the assembly of the mAb-coated beads into the trenches on the microchip. The antibody-coated nanobeads were somewhat larger than the commercially obtained PSL beads (ca. $320 \mathrm{~nm}$ ); the mAb 2C5-, mAb CEA-, and IgG-coated nanobeads had diameters of $360 \pm 3.3,324 \pm 8.3$, and $375 \pm 4.5 \mathrm{~nm}$, respectively (Fig.1b). The zeta potential of the uncoated beads was approximately $-70 \mathrm{mV}$. After adsorption of mAb 2C5, mAb CEA, and IgG, the zeta potentials were $-44.6 \pm 2.5,-59.5 \pm 0.5$, and $-38 \pm 2 \mathrm{mV}$, respectively (Fig.1c). The nanobeads did not aggregate (Fig.1d) and remained stable in terms of their sizes and zeta potentials. Prior to assembly, we used indirect ELISA to evaluate the activity of the adsorbed antibody (Fig.1e, using $\mathrm{mAb} 2 \mathrm{C} 5$ as an example). When compared with free mAb 2C5, the mAb $2 \mathrm{C} 5$ moieties adsorbed on the PSL nanobeads retained their anti-NS activity. The IgG-coated PSL nanobeads exhibited no specificity toward NS and the unmodified PSL nanobeads displayed no cross-reactivity to NS, confirming the specificity of the mAb 2C5-coated PSL nanobeads toward the cancer-related NS antigen. Similar retention of activity of immobilized mAb $2 \mathrm{C} 5$ on the surfaces of liposomes and polymeric micelles has been reported earlier 24,25 .

\section{Biosensor Fabrication and Selective assembly of nanobeads on biochips}

We designed the biosensors such that it was small enough to fit on a cylindrical microscale catheter (Fig. 2a). The biosensors were placed onto a millimeter-scale holder such that an end effector could hold it for

precise configuration and maneuvering. Trenches were formed using electron beam lithography ${ }^{26}$. The antibody-PSL nanobeads were assembled on each chip electrophoretically, as presented schematically in Fig.2b, using the instrument setup displayed in Fig.2c. We fabricated the biosensor with multiple active and separate trench areas of each $70 \mu \mathrm{m}^{\prime} 70 \mu \mathrm{m}$ each. Because these trench areas were isolated from each other, with the help of electrophoresis we could selectively assemble different NPs coated with 
specific antibodies in a specific trench that could aid in simultaneous multiple detection of different biomarkers. For instance, we have previously described the assembly of 100 - and $60-\mathrm{nm}$ red fluorescent PSL NPs, on to three out of four regions of the biosensor ${ }^{27}$. However, for this study we assembled 320nm CEA antibody-coated PSL NPs. The nanobeads were assembled on each microchip to cover greater than $90 \%$ of the trench area, with no aggregation (Fig.2d).

\section{In vitro and in vivo testing}

Following assembly, the mAb $2 \mathrm{C} 5$ biochips were tested for their ability to interact with fluorescently labeled NS using a direct "ELISA-like" assay. The fluorescence intensities of the mAb 2C5 biochips were greater than that of the control IgG biochip in a concentration-dependent manner (Fig.3a), confirming the specificity of the mAb $2 \mathrm{C} 5$ biochips toward NS, in agreement with the indirect ELISA data in the plate format. Here, the fluorescence intensity of the IgG biochips was considered to represent the background signal. Image $\mathrm{J}$ analysis revealed that the mAb 2C5 nanobeads assembled on the chips were able to detect NS specifically at low concentrations in FBS (Fig.3b). Clinically, high levels of NS are detected in individuals suffering from diverse forms of cancer ${ }^{28}$. Normal NS levels of $16 \pm 8.8 \mathrm{ng} / \mathrm{mL}$ (range $8-52$ $\mathrm{ng} / \mathrm{mL}$ ) have been reported using the traditional ELISA method ${ }^{29}$. With the microchips, we could detect NS antigen at concentrations down to $62.5 \mathrm{pg} / \mathrm{mL}$ in FBS, suggesting that this biosensor is much more sensitive than a conventional 96-well plate assay.

We confirmed the applicability of our device for the detection of CEA biomarkers using a classic sandwich ELISA technique (Fig.4a). Initial tests with CEA were carried out in vitro, to study the detection of the antigen in PBS, FBS, and 50\% murine blood. Although the CEA biochips demonstrated a very high sensitivity in PBS and FBS, with the ability to detect antigen at concentrations down to $31.3 \mathrm{pg} / \mathrm{mL}$ (data not shown), the sensitivity toward the antigen in $50 \%$ murine blood was even higher, with detection at as low as $15.6 \mathrm{pg} / \mathrm{mL}$ [Fig.4b (upper panel) and Fig.4c]. Notably, we could use the microchip system to detect CEA, at a detection limit of $31.3 \mathrm{pg} / \mathrm{mL}$, in blood samples obtained from antigen-spiked mice [Fig.4B (lower panel) and Fig.4d]. These levels of detection are much lower than those provided by commercially available CEA ELISA kits ${ }^{30}$ or by a quantum dot-based nano-bio-chip system ${ }^{31}$, confirming the high sensitivity of our system.

We further evaluated the sensitivity of our microchip by simulating in vivo pathology conditions. Tests run on CEA biochips with blood samples from tumor-bearing mice revealed increased fluorescence from day 1 to day 7 relative to the background (Fig.5a). Following quantification, a significant increase in the intensities occurred at day 7 (Fig.5b), suggesting that higher levels of CEA were present in the circulation, indicating tumor growth. The mice exhibited no visible signs of tumor at days 1 and 3 , but clear detectable vascularized tumors were observed by day 7. Nude mice with colonic-tumor xenografts and tumor weights from $160 \mathrm{mg}$ to $2.16 \mathrm{~g}$ have been reported to possess serum CEA levels of less than 11.4 $\mathrm{ng} / \mathrm{mL}^{32}$. Thus, this biochip was sensitive to detect CEA in tumor-bearing mice at much lower concentrations-notably, concentrations corresponding to much earlier stages of tumor growth. Under such conditions, the commercial CEA ELISA kit could not detect CEA (Fig.5c). 
PSL nanobeads presenting adsorbed antibodies can be assembled onto a biochip to provide a convenient biosensor for the detection of various biomarkers. These biosensors can detect very low concentrations of cancer biomarkers, such as NS and CEA, in various media, including blood from tumorbearing animals. The main advantages of such a system are simplicity of preparation and high sensitivity; such chips might be useful for the simultaneous detection of several distinct biomarkers in biological fluids and for the early detection of cancer (or other diseases) and control of its progression and treatment. It would also be possible to modify the manufacturing process to control the number of trenches and to assemble nanobeads with different adsorbed antibodies in different trenches to allow the simultaneous detection of more than one biomarker in a very small biological sample. Using this system, any biomarker for an existing ELISA setup could be detected with high sensitivity and relatively low cost.

\section{Discussion}

\section{Nanobead-antibody immobilization}

The adsorption of $A b$ on the PSL nanobeads is a result of the capacity of the $\lg G$ molecule to bind to the PSL nanobeads and the density and surface area of the PSL nanobeads itself. Unlike covalent conjugation ${ }^{33-35}$, adsorption is simple, does not require additional chemical reagents, and does not result in aggregation. For adsorption of a monolayer of antibody on the surface of the nanobeads with correct spatial orientation and decreased likelihood of nonspecific binding, the amount of antibody needed was calculated based on the following formula provided by Bangs Laboratory (Fishers, IN, USA) and also suggested by 22,23 .

$S=(6 / \rho D)(C)$

Where

$\mathrm{S}=$ amount of representative protein needed to achieve surface saturation ( $\mathrm{mg}$ protein $/ \mathrm{g}$ of microspheres), $\mathrm{C}=$ capacity of microsphere surface for a given protein, which will vary depending on the size and molecular weight of the protein to be coupled ( $\mathrm{mg}$ protein $/ \mathrm{m}^{2}$ of polymer surface). For lgG type antibodies $C=2.5 \mathrm{mg} / \mathrm{m}^{2}, 6 / \rho D=$ surface area/mass $(\mathrm{m} 2 / \mathrm{g})$ for microspheres of a given diameter $(\rho=$ density of microspheres, which for polystyrene is $\left.1.05 \mathrm{~g} / \mathrm{cm}^{3}\right)$, and $D=$ diameter of microspheres, in microns.

Based on equation (1), a monolayer antibody molecule was adsorbed on the PSL nanobeads. Other methods of conjugation chemistry are described ${ }^{33-35}$ using agents such as EDC. However, adsorption techniques require no additional reagents and are a simple technique resulting in no aggregation. In addition, the nanobeads were stable in terms of their size and zeta potential, which is a critical factor in the assembly of antibody-coated nanobeads into the nano-trenches (Figure). 


\section{Biosensor Fabrication}

We fabricated these biosensors on polished 3-inch silicon wafers using optical lithography and E-Beam lithography with desired nano features or trenches (10 micron apart) on Poly(methyl methacrylate) (PMMA) that is spun on the gold substrates with a film thickness of $150 \mathrm{~nm}$. Compared to the current multi-level masking semiconductor techniques, our approach is relatively simple. To have a successful assembly of the antibody coated NPs, we had to ensure that the PMMA was secured to the gold layer underneath. During our lithography process, after spinning the PMMA layer we baked the wafer at $180^{\circ} \mathrm{C}$ for 30 minutes. However, due to the glass transition temperature for PMMA being $105^{\circ} \mathrm{C}$, we altered our baking process by covering the substrate and baked it at $100^{\circ} \mathrm{C}$ for 30 minutes. As a result, the PMMA adhered better to the gold layer.

As an alternate option SU-8 based biosensors can also be used which is more cost effective and pliable as compared to silicon wafers, however, fabrication of a smooth and flat template was a challenge. The non-flat surface (even with a variation of one micron over a several millimeters) does affect the e-beam lithography used to make the trenches as well as images the florescent sandwiched tags after antigen detection because of the variation of the depth of focus. At this point the biosensors were ready for assembly of the antibody-coated NPs.

\section{Assembly of antibody-coated nanobeads}

Assembly of NPs has gained attention due to potential applications in various emerging fields such as nanophotonic crystals, nanosensors and nanoelectronics. Herein, we developed a sequential template assisted electrophoretic assembly technique that can be utilized to assemble nanoparticles arrays over a large area and in short time. The electric force acting on the negatively charged colloidal nanoparticles depends on different variables including voltage and the distance between the electrodes. The time it takes for attaining full assembly coverage is dependent to the $\mathrm{pH}$ and the electric conductance of the nanoparticle solution and for our experimental conditions is between 2 and 3 minutes. Several parameters affect the electrophoretic assembly of NPs, such as time, voltage, zeta potential of the NPs and the concentration of NPs. Occasionally we would observe particle agglomeration during the optimization of the assembly process. The is a coulomb repulsion exerted on negatively charged NPs while in suspension; however, when the electrostatic force acting on the particles is large, suspended nanoparticles have enough energy to get close to the assembled particles and therefore the van der Waals forces can overcome the electrostatic repulsion which causes the agglomeration. This agglomeration was only observed with $\mathrm{CH}_{2} \mathrm{Cl}$ functionalized particles on top of the assembled patterns. Hence, we had to optimize the assembly parameters which control the electrostatic force during the assembly process to overcome particle agglomeration. Also adjusting the size and spacing of patterns is an important factor in preventing agglomeration by changing the electric field lines near the patterns. 
Uniform antibody coated-nanobeads together with its precise assembly is a contributing factor to increased sensitivity of out biosensor device.

\section{Testing of mAb2C5 and mAbCEA biochips}

Due to the specific activity of mAb against NS, we were able to detect and observe this binding in a concentration dependant fashion. High levels of NS are detected in individuals suffering from diverse forms of cancer, particularly lung cancer, breast cancer, renal and prostate cancer and lymphomas as a consequence of elimination systems that are overloaded or impaired in such disease states ${ }^{28}$. As a result, NS is a critical biomarker for additional detection and management of cancer. Normal levels of 16 $+/-8.8 \mathrm{ng} / \mathrm{ml}$, range $8-52 \mathrm{ng} / \mathrm{ml}$ were reported using the traditional ELISA method ${ }^{29}$, in comparison to which we were able to detect down to $62.5 \mathrm{pg} / \mathrm{ml}$ in FBS, suggesting that our bio-sensor was much more sensitive than the conventional 96 -well plate assay format.

Similarly, the CEA biomarker was also tested using sandwich ELISA. Low levels of CEA antigen were detected in PBS pH 7.4 and FBS at $37^{\circ} \mathrm{C}$. The limit of detection using the biochip was $31.25 \mathrm{pg} / \mathrm{ml}$ (data not shown). This is of great advantage considering the baseline levels of CEA are $2.5 \mathrm{ng} / \mathrm{ml}$ to $5 \mathrm{ng} / \mathrm{ml}$. Detecting low amounts of CEA in very early stages of cancer can help in better prognosis and effective treatment. Similar results were reported when CEA was detected in saliva using quantum dots based on the nano-bio-chip system ${ }^{31}$. In comparison, $15.6 \mathrm{pg} / \mathrm{ml}$ of CEA in spiked $50 \%$ murine blood was detected in vitro, suggesting our system was highly sensitive.

Additionally, the detection of CEA was studied ex vivo from antigen injected mice in a dose-dependent manner with a limit of detection down to $31.25 \mathrm{pg} / \mathrm{ml}$. These values were less than baseline levels and can be an advantage to detect minute changes in the biomarker levels during cancer development.

Further, the sensitivity of the nanochip was evaluated by simulating in vivo pathology conditions. Tests run with blood samples from tumor-bearing mice on CEA-biochips showed increased fluorescence from day 1 to day 7 compared to the background. Previous studies in nude mice with colonic-tumor xenografts and tumor weights from $160 \mathrm{mg}$ to $2.16 \mathrm{~g}$ reported serum CEA levels of less than $11.4 \mathrm{ng} / \mathrm{ml}^{32}$. The biochip was sensitive to detect the CEA antigen in tumor-bearing mice in much lower quantities, i.e. at much earlier stages of tumor growth even before a vascularized form of tumor was established. This is essentially important in early identification of tumor biomarkers in cases were biomarkers are undetected due to high cut-off values ${ }^{36}$ or in aggressive form of disease such as cancer ${ }^{37}$. We believe our highly sensitive biosensor will prove beneficial in identifying the biomarkers above mentioned conditions.

\section{Materials And Methods}

\section{Materials}


Carboxy-functionalized polystyrene nanobeads (PSL, 5\% w/v) were purchased from Sperotech (Lake Forest, IL). Nucleosomal antigen (calf thymus) was purchased from Worthington Biochemical (Lakewood, NJ). mAb 2C5 was produced by Harlan Bioproducts (Indianapolis, IL) using a hybridoma cell line from our laboratory 3837575655555555 . Clarified mouse myeloma ascites IgG-2a (kappa) UPC10 antibody was purchased from MP Biochemicals (Solon, $\mathrm{OH}$ ). Poly--lysine and fetal bovine serum (FBS) were obtained from Sigma-Aldrich (St. Louis, MO). Goat anti-mouse IgG peroxidase-conjugate was obtained from ICN Biochemicals (Aurora, $\mathrm{OH}$ ). K-blue TMB peroxidase substrate was purchased from Neogen Corporation (Lexington, KY). Oregon Green ${ }^{\circledR} 488$ carboxylic acid succinimidyl ester-5-isomer was purchased from Molecular Probes (Eugene, OR). The BCA protein assay kit was purchased from Pierce Biotechnology (Rockford, IL). All other chemicals and reagents were analytical-grade preparations from Sigma-Aldrich. Mouse monoclonal anti-CEA antibody [1C7], CEA protein, and secondary fluoresceinlabeled goat polyclonal antibody to mouse IgG-H\&L were obtained from Abcam (Cambridge, MA). Female Balc/C mice, ages 8-10 weeks, were purchased from Charles River Laboratory (Wilmington, MA).

\section{Antibody immobilization on PSL nanobeads and protein determination}

PSL nanobeads were coated with IgG, mAb 2C5, or anti-CEA mAb by adding $1.56 \mathrm{mg}$ of an antibody to 10 $\mathrm{mg}$ of a $1 \%(\mathrm{w} / \mathrm{v})$ suspension of the nanobeads in a total volume of $1 \mathrm{~mL}$ of PBS $(\mathrm{pH} 7.4)$. After incubation overnight at room temperature with gentle shaking, the suspension was centrifuged ( $1.5^{\prime}$ $1000 \mathrm{rpm}, 15 \mathrm{~min}$ ). The supernatant was removed and the concentration of the free antibody in it was determined using the BCA assay (according to the provider's instructions). The pellet was re-suspended in deionized (DI) water or PBS ( $\mathrm{pH} 7.4)$ and stored at $4{ }^{\circ} \mathrm{C}$.

\section{Particle-size and zeta potential}

The sizes and zeta potentials of all of the nanobead preparations were determined through dynamic light scattering and zeta phase light scattering, respectively, using a ZetaPlus particle size analyzer (Brookhaven Instrument, Holtsville, NY). The antibody-nanobead suspension was diluted with DI water for size measurements and with $1 \mathrm{mM} \mathrm{KCl}$ for zeta potential measurements. All analyses were repeated in triplicate.

\section{Immunological activity of mAb 2C5 PSL nanobeads determined through indirect ELISA}

96-well plates were incubated with poly-l-lysine $(40 \mathrm{mg} / \mathrm{mL}, 50 \mathrm{~mL})$ overnight at $4{ }^{\circ} \mathrm{C}$. Following incubation, the poly-l-lysine solution was discarded and the wells were blocked with Tris-buffered saline $(200 \mathrm{~mL})$ containing Tween 20 (TBST; 0.05\%, w/v) and casein (TBST-casein; $2 \mathrm{mg} / \mathrm{mL}$ ) for $1 \mathrm{~h}$ at room temperature (RT). After blocking, the wells were washed three times with TBST and incubated with NS in 
TBST-casein $(40 \mathrm{mg} / \mathrm{mL}, 50 \mathrm{~mL})$ for $1 \mathrm{~h}$ at RT. The wells were washed three times with TBST and incubated ( $1 \mathrm{~h}, \mathrm{RT})$ with different concentrations of mAb 2C5 as a standard, clarified mouse myeloma ascites IgG-2a (kappa) UPC10 antibody as a control, and mAb 2C5-coated PSL nanobeads and IgG-2a (kappa)-coated PSL nanobeads in TBST-casein. After incubation, the wells were washed three times with TBST and incubated (1 h, RT) with $50 \mathrm{~mL} /$ well of a 1:5000 dilution of a goat anti-mouse IgG peroxidase conjugate in TBST-casein. The wells were washed three times again and incubated (for $15 \mathrm{~min}$ or until a blue color developed) with enhanced K-blue TMB peroxidase substrate $(100 \mathrm{~mL})$. The 96 -well plate was read (Labsystems Multiscan MCC/360 microplate reader installed with GENESISLITE Windows-based microplate software) at a wavelength of $620 \mathrm{~nm}$ with the reference filter at $492 \mathrm{~nm}$.

\section{Template fabrication and assembly of mAb-PSL nanobeads into chip trenches}

The nanochip template was fabricated by depositing layers of metals ( $6 \mathrm{~nm}$ of $\mathrm{Cr} / 40 \mathrm{~nm}$ of $\mathrm{Au}$ ) onto a 380- $\mu$ m-thick Si wafer coated with $150 \mathrm{~nm}$ of thermal $\mathrm{SiO}_{2}$. A 300-nm-thick poly (methyl methacrylate) (PMMA) film was spun onto the Au template and baked at $100^{\circ} \mathrm{C}$ for $90 \mathrm{~s}$. Trench array patterns were generated on the PMMA through electron beam lithography. After exposure, the PMMA film was developed in a 1:3 mixture of methyl isobutyl ketone (MIBK) and isopropyl alcohol (IPA) for $70 \mathrm{~s}$, followed by an IPA rinse for $20 \mathrm{~s}$ and a DI water rinse for $5 \mathrm{~min}$.

For their assembly in the trenches, the mAb-coated PSL nanobeads were diluted in DI water and then $\mathrm{NH}_{4} \mathrm{OH}$ was added to increase the $\mathrm{pH}$ and conductivity of the nanobead suspension. The nanobeads were assembled in the trenches electrophoretically at a DC voltage of 2-3 V. The assembled chips were stored in DI water prior to use.

\section{Detection of NS antigen by mAb $2 C 5$ nanobeads assembled on biochips}

After confirming the activity of the mAb $2 \mathrm{C} 5$ nanobeads toward NS, the mAb $2 \mathrm{C} 5$ biochips were incubated overnight in a $2 \%$ BSA solution at $4{ }^{\circ} \mathrm{C}$ to block nonspecific binding on their surfaces. The chips were washed with DI water and then incubated $\left(37^{\circ} \mathrm{C}, 1 \mathrm{~h}\right)$ with solutions of fluorescently labeled NS (concentrations from 500 to $62.5 \mathrm{pg} / \mathrm{mL}$ ) in FBS. The chips were washed again with DI water and stored in PBS prior to fluorescence imaging, with the images acquired analyzed using Image $\mathrm{J}$ software $(\mathrm{NIH}$, Bethesda, MD). Nonspecific IgG-biochips were employed as a control.

\section{Biochip-based ELISA of anti-CEA mAb}

A sandwich ELISA on biochips was applied to test the specific activity of the chips with anti-CEA mAb. CEA-bearing biochips were tested for their capacity to detect low concentrations of the CEA. The 
assembled biochips were incubated (overnight, $4^{\circ} \mathrm{C}$ ) in $2 \%$ BSA to block nonspecific binding and then they were washed with DI water to remove the BSA. The chips were then incubated ( $2 \mathrm{~h}, \mathrm{RT})$ with dilutions of the CEA (concentrations from 125 to $15.6 \mathrm{pg} / \mathrm{mL}$ ) in PBS (pH 7.4) or in FBS. CEA biochips incubated with plain solutions were employed as blanks. For testing the CEA in $50 \%$ murine blood samples (the same range of CEA dilutions were used), the biosensors were incubated $(2 \mathrm{~h})$ at $37^{\circ} \mathrm{C}$ to mimic physiological conditions. Sodium citrate (1\%) was used as an anticoagulant at a 10 -fold blood dilution (to normalize volume of blood obtained from mice). The chips were washed in water, incubated ( $2 \mathrm{~h}, \mathrm{RT})$ with a secondary FITC-labeled $\lg G$ antibody $(2 \mu \mathrm{g} / \mathrm{mL})$, washed to remove any traces of nonspecifically bound secondary antibody, and then stored in PBS (pH 7.4) at $4{ }^{\circ} \mathrm{C}$ until required for microscopic analysis. The study was carried out in compliance with the ARRIVE guidelines.

\section{ELISA with the blood of CEA-spiked mice}

All animal work was conducted according to relevant national and international guidelines and following the approval of the Northeastern University Institutional Animal Care and Use Committee (NU- IACUC) prior to the start of this study. The study was also carried out in compliance with the ARRIVE guidelines. CEA biochips were tested for detection of the CEA level in the blood of 6-8-week-old Balb/C mice spiked with known concentrations of the antigen. A solution $(100 \mu \mathrm{L})$ of CEA in PBS was injected via the tail vein. Each dose was calculated by considering two variables: (i) the dilution factor based on estimated mouse blood volume (ca. $2 \mathrm{~mL} /$ mouse) and (ii) the dilution in $0.9 \% \mathrm{NaCl}$ prior to incubation with chips. At 1-2 min post-injection, the blood was collected by cardiac puncture in sodium citrate. Blood samples were diluted (50:50, v/v) with $\mathrm{NaCl}$ to obtain final CEA concentrations ranging from 12.5 to $31.3 \mathrm{pg} / \mathrm{mL}$. The samples were then incubated with the chips as described in the previous paragraph.

\section{Detection of CEA in tumor-bearing mice}

Female Balb/c mice were inoculated with $4 \mathrm{~T} 1$ breast cancer cells (100,000 cells/mouse) suspended in

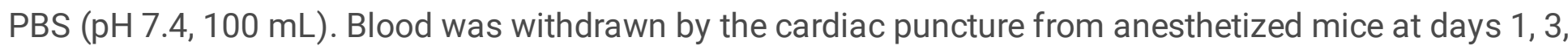
and 7. The blood samples $(0.3-0.7 \mathrm{~mL})$ were mixed with $50 \mu \mathrm{L}$ of $1 \%$ sodium citrate to prevent coagulation and stored at $4{ }^{\circ} \mathrm{C}$. CEA biochips were tested as mentioned earlier and then imaged and quantified. This study was also carried out in compliance with the ARRIVE guidelines.

\section{Imaging of biochips through fluorescence microscopy and image processing}

To quantify the detection of NS and CEA antigens, fluorescence images were processed using Image $\mathrm{J}$ software. Antibody-loaded biochips were imaged using a Nikon Optiphot 200 fluorescence microscope equipped with a charge-coupled device (CCD) camera. Images in both bright and dark fields (green 
channel fluorescence) were collected for each chip. Fluorescence imaging was performed using a 30-s exposure time with the CCD amplifier gain set to 0.6 ; images were stored as 24-bit jpg files. For processing, the background noise was removed through built-in background subtraction, followed by extraction of the 8-bit green color channel. The area of the image corresponding to trenches was manually selected and its mean fluorescence intensity analyzed. Similar treatments and analyses were performed for the images acquired from the control IgG biochips.

\section{Abbreviations}

mAb, monoclonal antibody; FBS, fetal bovine serum; PSL-COOH, carboxyl-functionalized polystyrene beads; BSA, bovine serum albumin; PBS, phosphate-buffered saline; NS, nucleosome; CEA, carcinoembryonic antigen; ELISA, enzyme-linked immunosorbent assay; PSA, prostate-specific antigen; CA 125, Cancer Antigen-125; CA 19-9, Carbohydrate antigen 19-9; AFP, alpha-fetoprotein.

\section{Declarations}

\section{Acknowledgments}

This study was supported by the W. M. Keck Foundation (grant no. 06-003599A00) and the National Science Foundation Nanoscale Science and Engineering Center (NSEC) for High-Rate Nanomanufacturing (grant no. 0832785). Experiments were conducted at the George J. Kostas Nanoscale Technology and Manufacturing Research Center at Northeastern University and with collaborators from the Center for Pharmaceutical Biotechnology and Nanomedicine.

\section{Author Contributions}

A.B. and V.P.T. conceived the idea. J.R.U. and T.M. designed the experiments. T.M. and J.R.U. performed the experiments. T.M. and J.R.U. analyzed the data. W.C.H. and T.L. contributed to the animal study. S.S., S.S., A.M., and C.Y. fabricated and assembled the biochips. S.S., A.M., and C.Y. acquired the images. All authors discussed the results. J.R.U and T.M. co-wrote the initial draft of the paper. W.C.H., A.B. and V.P.T. contributed editorial help.

\section{References}

1 Clark, L. C., Jr. \& Lyons, C. Electrode systems for continuous monitoring in cardiovascular surgery. Ann N Y Acad Sci 102, 29-45 (1962).

2 Halliwell, C. M., Simon, E., Toh, C. S., Bartlett, P. N. \& Cass, A. E. A method for the determination of enzyme mass loading on an electrode surface through radioisotope labelling. Biosens Bioelectron 17, 965-972, doi:S095656630200088X [pii] (2002). 
3 Chen, R. et al. Pancreatic cancer proteome: the proteins that underlie invasion, metastasis, and immunologic escape. Gastroenterology 129, 1187-1197, doi:S0016-5085(05)01579-9 [pii]

10.1053/j.gastro.2005.08.001 (2005).

4 Rahman, M. A., Kwon, N. H., Won, M. S., Choe, E. S. \& Shim, Y. B. Functionalized conducting polymer as an enzyme-immobilizing substrate: an amperometric glutamate microbiosensor for in vivo measurements. Anal Chem 77, 4854-4860, doi:10.1021/ac050558v (2005).

5 Srinivas, P. R., Kramer, B. S. \& Srivastava, S. Trends in biomarker research for cancer detection. Lancet Onco/ 2, 698-704, doi:S1470-2045(01)00560-5 [pii]

10.1016/S1470-2045(01)00560-5 (2001).

6 Verma, M. \& Srivastava, S. New cancer biomarkers deriving from $\mathrm{NCl}$ early detection research. Recent Results Cancer Res 163, 72-84; discussion 264-266 (2003).

7 Steinberg, W. The clinical utility of the CA 19-9 tumor-associated antigen. Am J Gastroentero/ 85, 350-355 (1990).

8 Catalona, W. J. et al. Measurement of prostate-specific antigen in serum as a screening test for prostate cancer. N Engl J Med 324, 1156-1161, doi:10.1056/NEJM199104253241702 (1991).

9 Holdenrieder, S. et al. Clinical relevance of circulating nucleosomes in cancer. Ann N Y Acad Sci 1137, 180-189, doi:NYAS1137012 [pii]

10.1196/annals.1448.012 (2008).

10 Gold, P. \& Freedman, S. O. Demonstration of Tumor-Specific Antigens in Human Colonic Carcinomata by Immunological Tolerance and Absorption Techniques. J Exp Med 121, 439-462 (1965).

11 De Potter, C. R., Beghin, C., Praet, M. M., Pauwels, C. F. \& Roels, H. J. CEA and HMFG in hyperplastic and malignant lesions of the breast. Pathol Res Pract 183, 271-276 (1988).

12 Zheng, G., Patolsky, F., Cui, Y., Wang, W. U. \& Lieber, C. M. Multiplexed electrical detection of cancer markers with nanowire sensor arrays. Nat Biotechnol 23, 1294-1301, doi:nbt1138 [pii]

10.1038/nbt1138 (2005).

13 Schweitzer, B. et al. Immunoassays with rolling circle DNA amplification: a versatile platform for ultrasensitive antigen detection. Proc Natl Acad Sci U S A 97, 10113-10119, doi:10.1073/pnas.170237197 170237197 [pii] (2000). 
14 Wu, G. et al. Bioassay of prostate-specific antigen (PSA) using microcantilevers. Nat Biotechnol 19, 856-860, doi:10.1038/nbt0901-856

nbt0901-856 [pii] (2001).

15 Nam, J. M., Thaxton, C. S. \& Mirkin, C. A. Nanoparticle-based bio-bar codes for the ultrasensitive detection of proteins. Science 301, 1884-1886, doi:10.1126/science.1088755

301/5641/1884 [pii] (2003).

16 Cheow, L. F., Ko, S. H., Kim, S. J., Kang, K. H. \& Han, J. Increasing the sensitivity of enzyme-linked immunosorbent assay using multiplexed electrokinetic concentrator. Anal Chem 82, 3383-3388, doi:10.1021/ac9024335 (2010).

17 Levenson, V. V. Biomarkers for early detection of breast cancer: what, when, and where? Biochim Biophys Acta 1770, 847-856, doi:S0304-4165(07)00040-2 [pii]

10.1016/j.bbagen.2007.01.017 (2007).

18 Taussig, M. J. \& Landegren, U. Progress in antibody arrays. TARGETS 2, 169-176 (2003).

19 Luo, X., Morrin, A., Killard, A. J. \& Smyth, M. R. Application of Nanoparticles in Electrochemical Sensors and Biosensors. Electroanalysis 18, 319-326, doi:10.1002/elan.200503415 (2006).

20 Brogan, K. L., Wolfe, K. N., Jones, P. A. \& Schoenfisch, M. H. Direct oriented immobilization of F(ab') antibody fragments on gold. Analytica Chimica Acta 496, 73-80 (2003).

21 de Wildt, R. M., Mundy, C. R., Gorick, B. D. \& Tomlinson, I. M. Antibody arrays for high-throughput screening of antibody-antigen interactions. Nat Biotechno/ 18, 989-994, doi:10.1038/79494 (2000).

22 Bale, M. D., Danielson, S. J., Daiss, J. L., Goppert, K. E. \& Sutton, R. C. Influence of copolymer composition on protein adsorption and structural rearrangements at the polymer surface. Journal of Colloid and Interface Science 132, 176-187 (1989).

23 Cantarero, L. A., Butler, J. E. \& Osborne, J. W. The adsorptive characteristics of proteins for polystyrene and their significance in solid-phase immunoassays. Analytical Biochemistry 105, 375-382 (1980).

24 Torchilin, V. P. Targeted pharmaceutical nanocarriers for cancer therapy and imaging. The AAPS journal 9, E128-147 (2007).

25 Elbayoumi, T. A., Pabba, S., Roby, A. \& Torchilin, V. P. Antinucleosome antibody-modified liposomes and lipid-core micelles for tumor-targeted delivery of therapeutic and diagnostic agents. $J$ Liposome Res 17, 1-14, doi:777389336 [pii] 
10.1080/08982100601186474 (2007).

26 Siavoshi, S. et al. Size-selective template-assisted electrophoretic assembly of nanoparticles for biosensing applications. Langmuir 27, 7301-7306, doi:10.1021/la104975u (2011).

27 Malima, A. et al. Highly sensitive microscale in vivo sensor enabled by electrophoretic assembly of nanoparticles for multiple biomarker detection. Lab Chip 12, 4748-4754, doi:10.1039/c2lc40580f (2012).

28 Holdenrieder, S. et al. Nucleosomes in serum as a marker for cell death. Clin Chem Lab Med 39, 596-605, doi:10.1515/CCLM.2001.095 (2001).

29 Amoura, Z. et al. Circulating plasma levels of nucleosomes in patients with systemic lupus erythematosus: correlation with serum antinucleosome antibody titers and absence of clear association with disease activity. Arthritis Rheum 40, 2217-2225, doi:10.1002/1529-0131(199712)40:12\&lt;2217::AIDART16\&gt;3.0.CO;2-P (1997).

30 Dungchai, W. et al. Development of a sensitive micro-magnetic chemiluminescence enzyme immunoassay for the determination of carcinoembryonic antigen. Analytical and Bioanalytical Chemistry 387, 1965-1971, doi:10.1007/s00216-006-0899-y (2007).

31 Jokerst, J. V. et al. Nano-bio-chips for high performance multiplexed protein detection: determinations of cancer biomarkers in serum and saliva using quantum dot bioconjugate labels. Biosens Bioelectron 24, 3622-3629, doi:S0956-5663(09)00284-X [pii]

10.1016/j.bios.2009.05.026 (2009).

32 Lewis, J. C. \& Keep, P. A. Relationship of serum CEA levels to tumour size and CEA content in nude mice bearing colonic-tumour xenografts. Br J Cancer 44, 381-387 (1981).

33 Dhawan, S. Design and construction of novel molecular conjugates for signal amplification (II): use of multivalent polystyrene microparticles and lysine peptide chains to generate immunoglobulinhorseradish peroxidase conjugates. Peptides 23, 2099-2110, doi:S019697810200253X [pii] (2002).

34 Blanchette, C. D. et al. Decoupling internalization, acidification and phagosomalendosomal/lysosomal fusion during phagocytosis of InlA coated beads in epithelial cells. PLoS One 4, e6056, doi:10.1371/journal.pone.0006056 (2009).

35 Kim, M., Yun, C. H., Park, S. K., Seo, J. H. \& Ahn, T. Production of polyclonal antibodies against peptide antigens using polystyrene beads as a carrier. Biotechnol Lett 29, 1735-1740, doi:10.1007/s10529-007-9463-x (2007).

36 Wang, W. S. et al. Preoperative carcinoembryonic antigen level as an independent prognostic factor in colorectal cancer: Taiwan experience. Jpn J Clin Oncol 30, 12-16 (2000). 
10.1016/S0140-6736(98)07127-X (1999).

38 lakoubov, L. Z. \& Torchilin, V. P. A novel class of antitumor antibodies: nucleosome-restricted antinuclear autoantibodies (ANA) from healthy aged nonautoimmune mice. Oncol Res 9, 439-446 (1997).

\section{Figures}
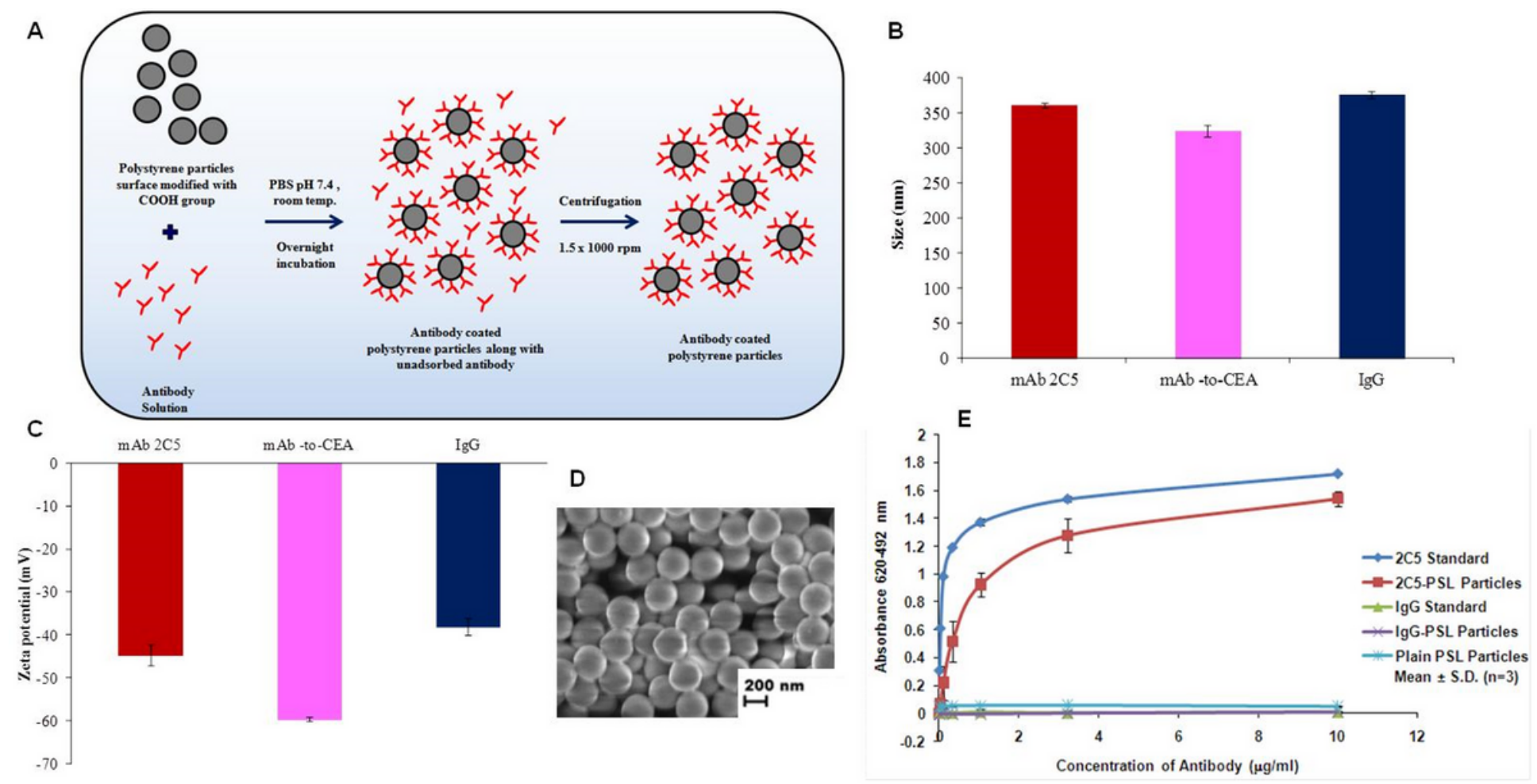

Figure 1

Preparation and characterization of antibody-coated nanoparticles. Carboxyl polystyrene nanobeads (PSL) were coated with IgG, monoclonal antibody mAb 2C5, or mAb CEA (a). The antibody-coated particles were separated from the free antibody through centrifugation and then characterized for their particle size (b) and zeta potential (c). Under a scanning electron microscope, the particles revealed no aggregation following antibody coating (d). When compared with standard free mAb 2C5, the mAb 2C5coated PSL particles retained their specific activity toward NS. Controls (IgG standard, IgG PSL particles, and plain PSL particles) exhibited no activity (e). $(n=5$, mean \pm S.D.). 


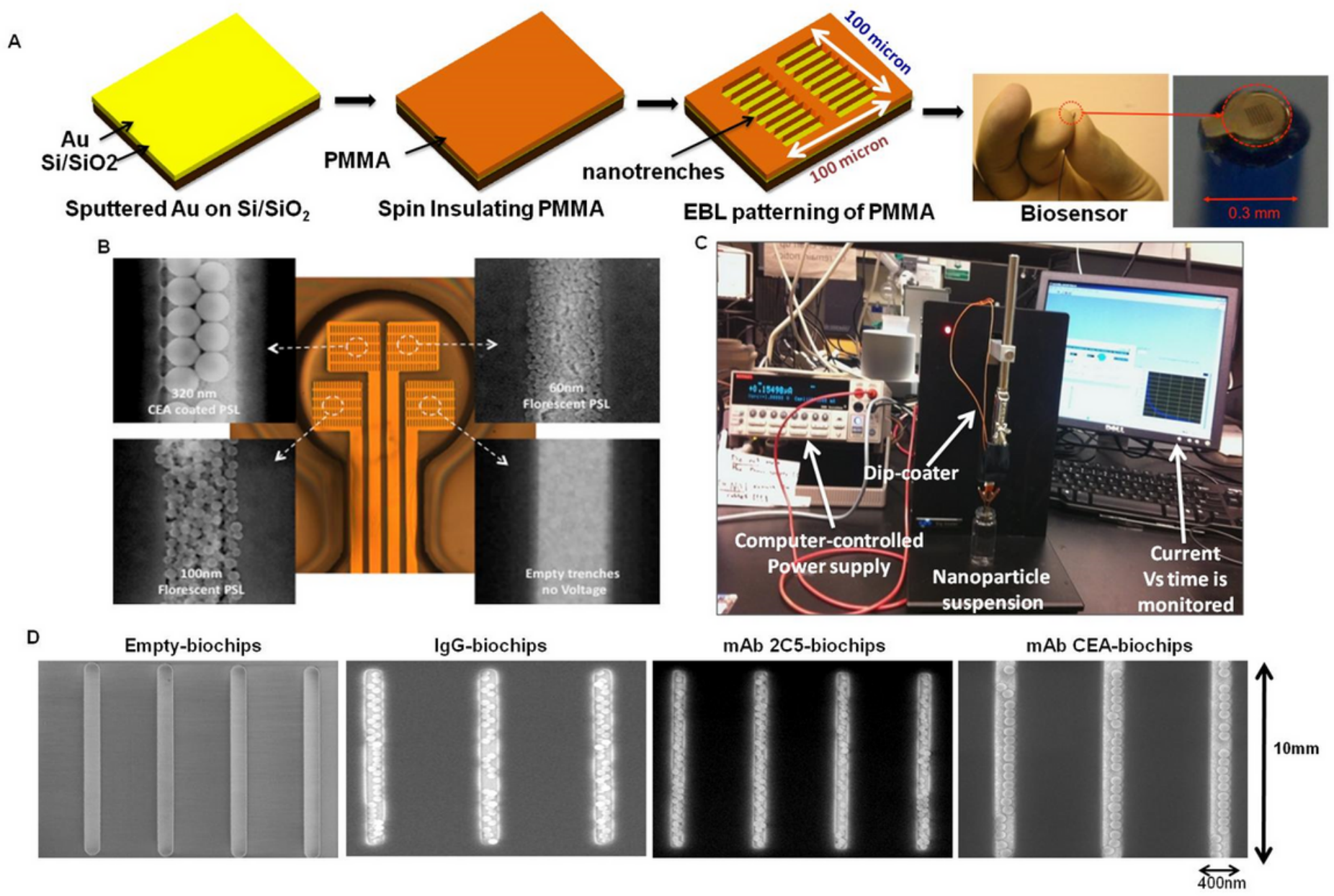

Figure 2

Template fabrication and instrumentation setup for nanobead assembly onto a microchip Trenches were created in surface PMMA films using electron beam lithography (a). Optical and SEM images of a biosensor with four electrically separated regions in which electrophoretic assembly was performed individually to assemble various NPs. (b). A particle suspension at pH 10.7-11.1 and a DC voltage of 2$3 \mathrm{~V}$ were used for a period of 60-180 s for assembly in the instrumental setup (c). Following assembly of the antibody-coated particles in 400-nm-wide, 10- $\mu \mathrm{m}$-long trenches, SEM images revealed assembly without particle aggregation (d). 
A

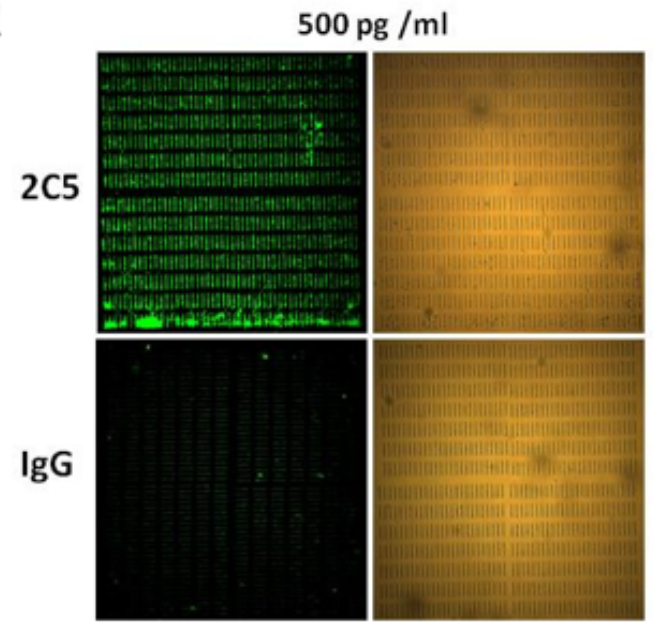

$125 \mathrm{pg} / \mathrm{ml}$

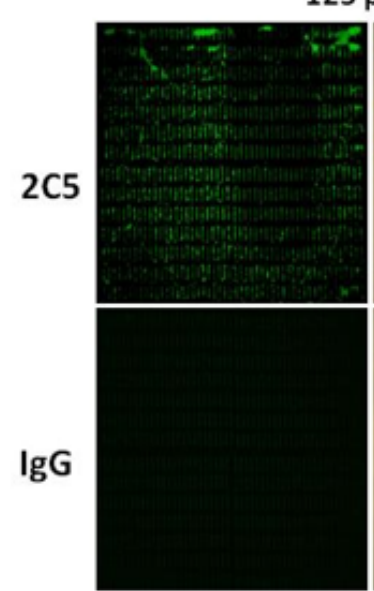

Dark Field

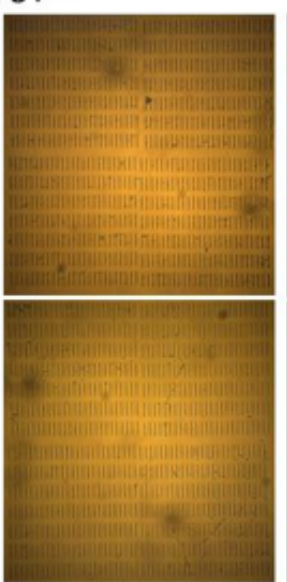

Bright Field

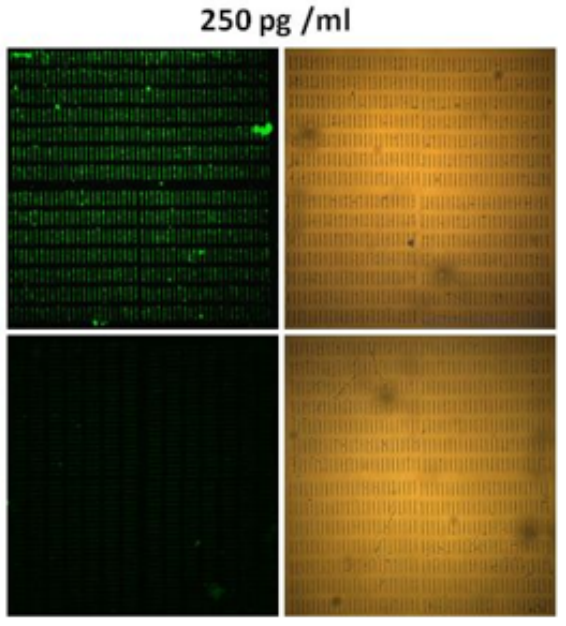

$62.5 \mathrm{pg} / \mathrm{ml}$

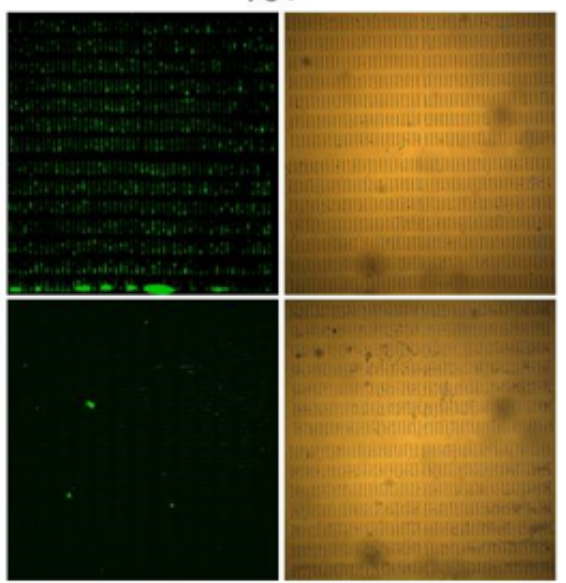

Bright Field

B

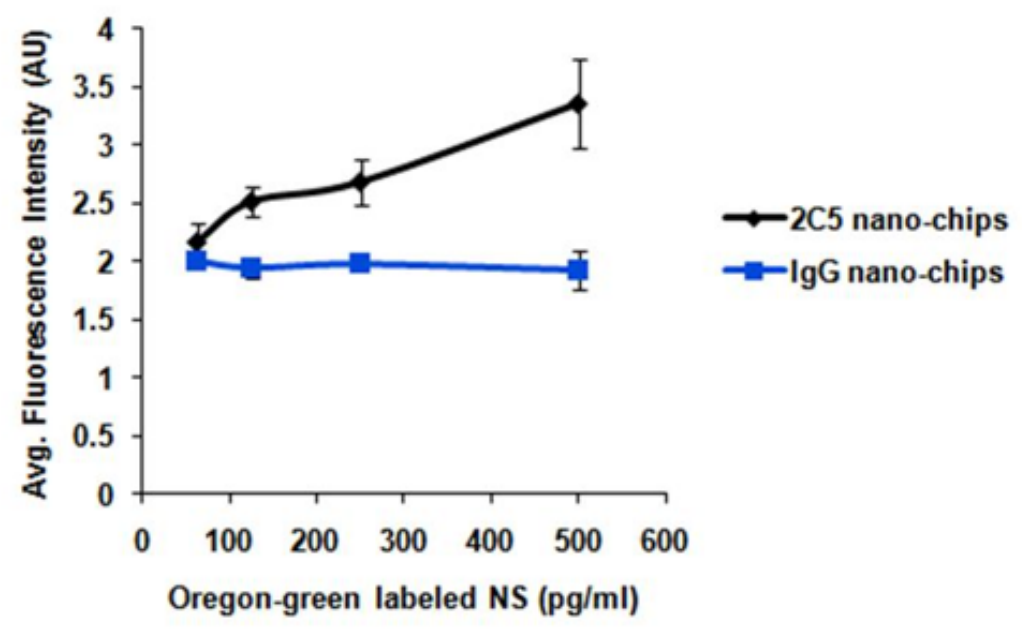

\section{Figure 3}

Indirect detection of NS using mAb2C5 biochips. The mAb $2 \mathrm{C} 5$ beads were assembled on chips and incubated with different concentrations of Oregon Green ${ }^{\circledR}$ labeled-NS in FBS and compared with the same antigen dilutions on IgG biochips. Fluorescence images revealing the presence of NS on the mAb $2 \mathrm{C} 5$ biochips (a) and corresponding quantification of data obtained using Image $\mathrm{J}$ software $(\mathrm{b})$. $(\mathrm{n}=3$; mean \pm S.D.). 

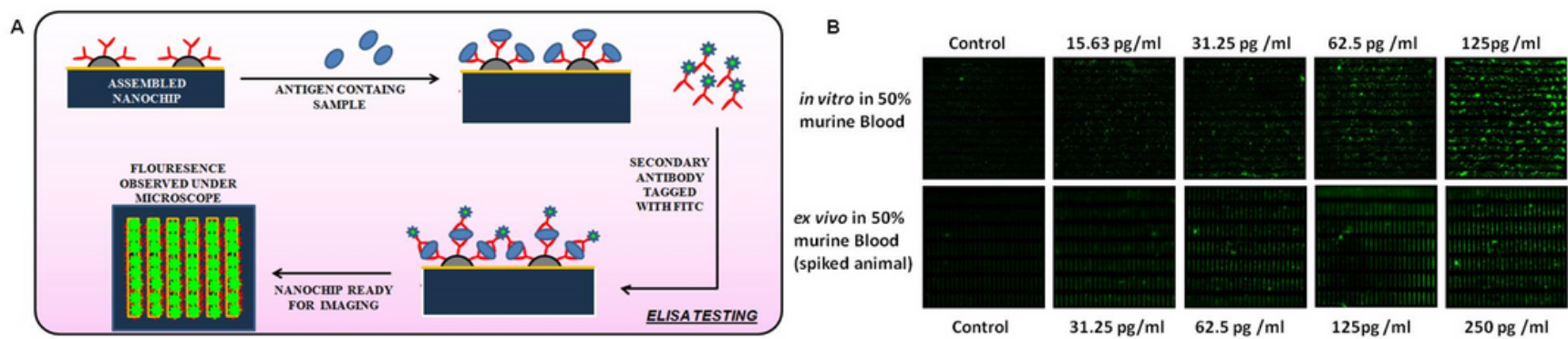

Control

$31.25 \mathrm{pg} / \mathrm{ml} \quad 62.5 \mathrm{pg} / \mathrm{ml}$

$125 \mathrm{pg} / \mathrm{ml}$

$250 \mathrm{pg} / \mathrm{ml}$

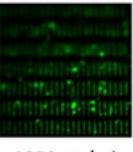

D
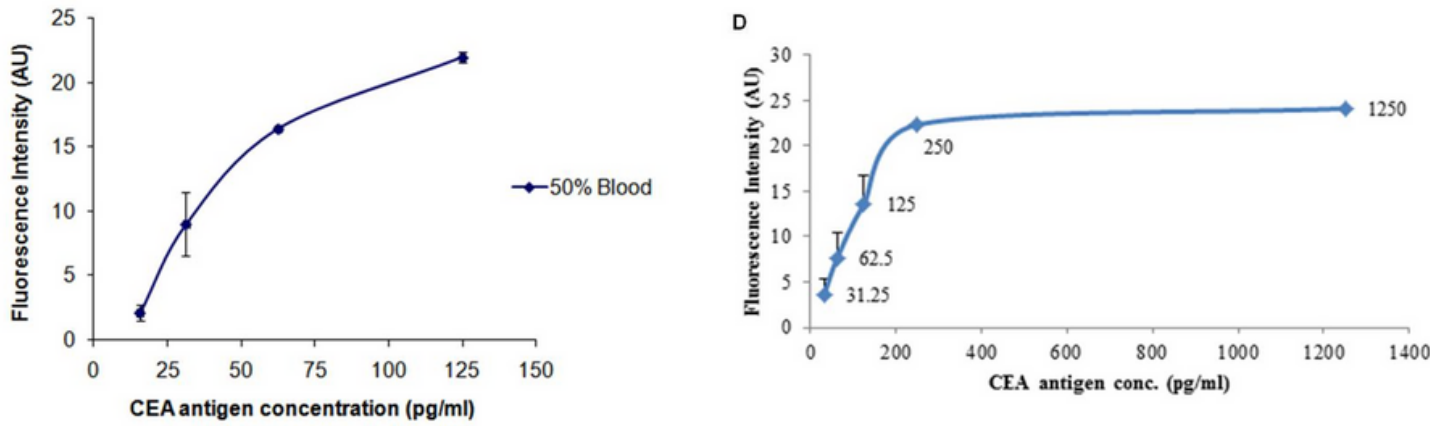

$1250 \mathrm{pg} / \mathrm{ml}$

c

\section{Figure 4}

In vitro and ex vivo ELISA on mAb CEA biochips In vitro testing of CEA biochips was performed using the sandwich ELISA technique (a). Signal amplification was achieved using a secondary antibody tagged with a fluorophore. Fluorescence images of CEA biochips detecting different concentrations of CEA in vitro (upper panel) and ex vivo (lower panel) in blood samples spiked with CEA (b). Images were quantified using Image $\mathrm{J}$ software; fluorescence intensities plotted with respect to CEA concentration for in vitro (c) and ex vivo (d) data. ( $n=3$; mean \pm S.D.). 
A

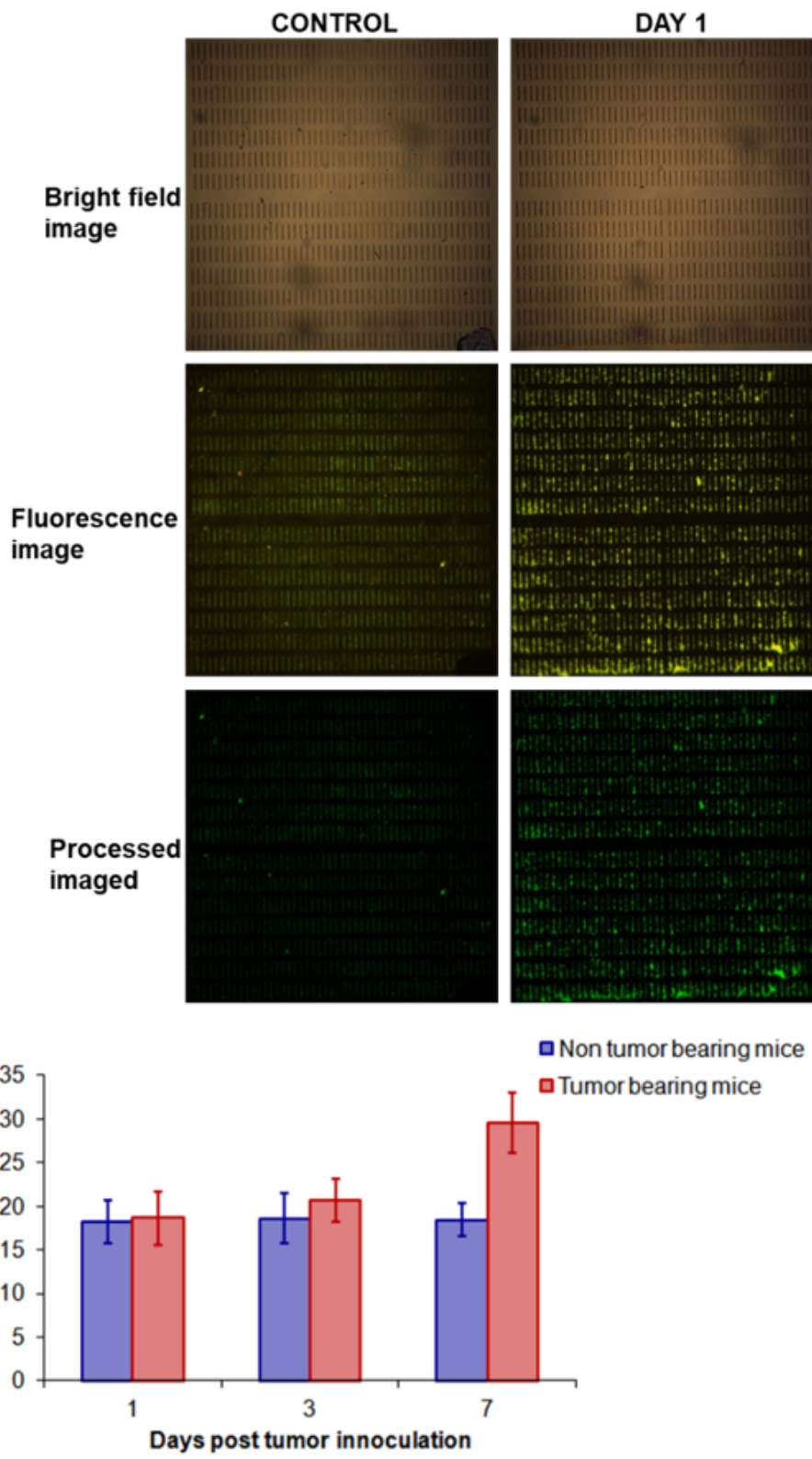

DAY 3
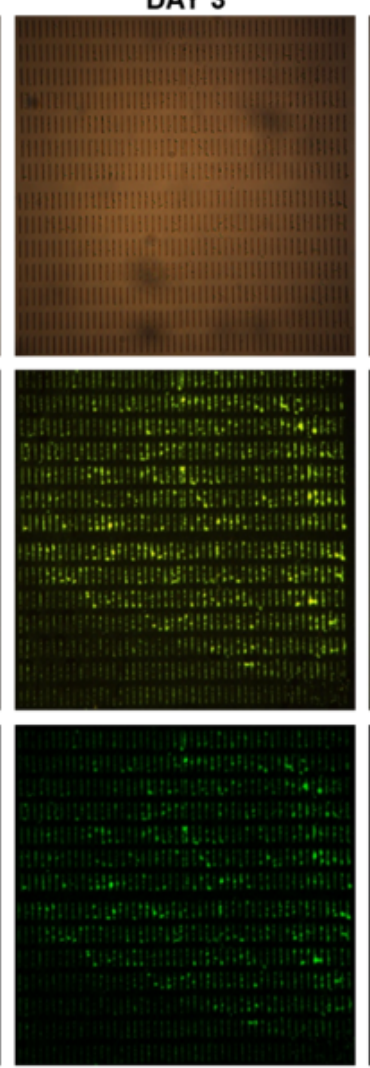

C

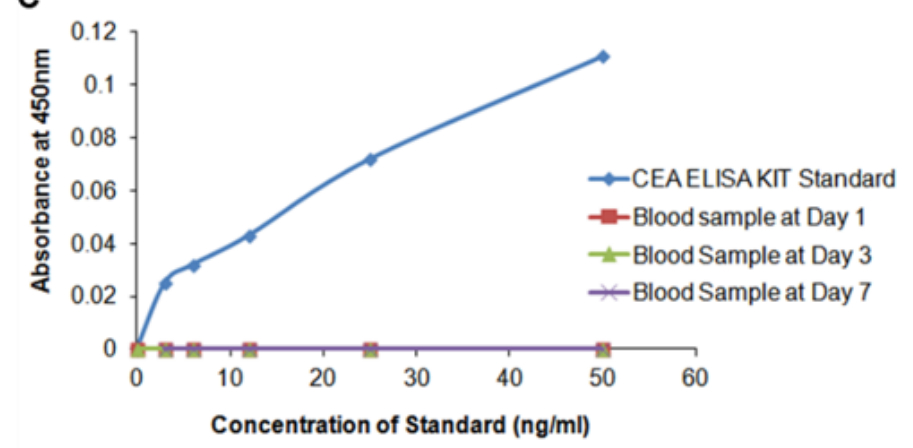

\section{Figure 5}

ELISA testing of blood from tumor-bearing mice on mAb CEA biochips Blood samples from tumor-bearing mice were tested on CEA biochips, using a sandwich ELISA technique, on days 1, 3, and 7 postinoculation; fluorescence images were recorded (a). Signals from the images were quantified using Image $\mathrm{J}$ software; fluorescence intensity is represented in arbitrary units (b). Blood samples were also tested for CEA using a commercially available CEA kit (c). ( $n=3$ chips; mean \pm S.D.). 